\title{
Quantified Comparison of Uterine Electrical Activity during Active Labor at various locations in Abdominal Surface: A Preliminary Study
}

\author{
Santosh N Vasist ${ }^{1}$, Dr. Parvati Bhat ${ }^{2}$, Dr. Shrutin Ulman ${ }^{3}$, Dr. Harishchandra Hebbar ${ }^{4}$ \\ ${ }^{1}$ PhD Research scholar, Manipal School of Information Sciences (MSOIS), Manipal Academy of Higher \\ Education (MAHE), India, santoshvasist@gmail.com \\ ${ }^{2}$ Professor, Melaka Manipal Medical College (MMMC), Manipal Academy of Higher Education (MAHE), \\ parvati.bhat@manipal.edu, \\ ${ }^{3}$ Senior Principal Scientist, Philips Healthcare, India, shrutin.ulman@philips.com \\ ${ }^{4}$ Professor, Manipal School of Information Sciences (MSOIS), Manipal Academy of Higher Education
}

(MAHE), India, harish.hebbar@manipal.edu

\begin{abstract}
Electrohysterography or the study of the electrical propagation patterns of the uterine contractile activity when combined with modern computing capabilities can prove to be a powerful tool in obstetric care. In this preliminary study the electrical activity during labor contractions was compared quantitatively at various locations on the abdominal surface. Data was collected from 10 participants for about 30 minutes using 7 electrodes mono-polar configuration. The electrical activity was found to be concentrated along the umbilical axis. The center of the electrical activity was found to be non-stationary and move from the lower to the upper uterine segment as the contraction intensified. The quantified electrical activity at various locations may serve as a template for placement of electrodes for future studies.
\end{abstract}

Key words: uterine electrohysterography, uterine contractions, labour

\section{INTRODUCTION}

Uterine contractions play a crucial role in both term and preterm labour. Early detection of preterm labour and non-progress in full term labour can lead to effective treatment plans to improve medical outcomes. The electrical activity arising in the myometrium of the uterus muscle has been widely accepted to be associated with uterine contractions [1]-[3]. The study of electrical signals from the uterus muscles is also popularly called as 'Electrohysterography' (EHG) and modern techniques of EHG is non-invasive. Studies have shown EHG to be better than existing techniques such as CTG (cardiotocography) and safer than IUPC (intra uterine pressure catheter) [4]. The analysis of EHG signals can reveal patterns regarding the activity of the uterus that can be used for early detection of preterm labor [5], [6]. Studies have also attempted at predicting the risk of non-progress of labour in term pregnancies based on the analysis of EHG signals [7]-[9].

Uterus muscle is composed of millions of myometrial cells. The flow of electrical signals in these myometrial cells initiates and sustains uterine contractions. This process of initiating a contraction is similar to other muscle tissues, however uterus is able to contract without nervous or hormonal inputs [10]. These electrical signals are referred to as actionpotentials or spikes. Electrical activity in the myometrium is a result of the de-polarization and repolarization of the smooth muscle cells. Depolarization of one cell leads to the activation of a neighboring non de-polarized cell, in turn initiating a wave of activation in a specific direction [11].

The depolarization phase of the action potential is caused by an inward current carried by the $\mathrm{Ca} 2+$ and $\mathrm{Na}+$ ions in the uterus muscle. The repolarization phase in the action potential is caused by an outward current carried by $\mathrm{K}+$ ions [12]. Uterine contractions are stimulated or suppressed by modifying the electrical properties of the myometrial cells.

EHG signals can be characterized as action potentials fast wave having amplitude modulated by the slow wave. This slow wave corresponds to the mechanical signal and can be regarded as the contractions wave. This concept is called as amplitude modulation [13]. The reverse is also possible, i.e., the separation of either the slow wave or the fast wave from the complete EHG signal and is called as amplitude demodulation. The frequency range of slow wave is 0.005 to $0.3 \mathrm{~Hz}$, while it is 0.1 to $3 \mathrm{~Hz}$ for the fast wave [14]. Amplitude demodulation can be achieved by using filtering technique [13], [14]. While many methods have been employed to visualize the slow wave from the EHG signal, filtration method 
provided stronger averaging and attenuation of amplitudes of action potentials [13].

\section{METHODOLOGY}

The objective of this preliminary study was to quantitatively compare the level of electrical activity in the uterus during active labour at various locations on the abdominal surface. In this study, we collected EHG data from 10 pregnant women in active labour. The institutional ethics committee approved the protocol for this study, and signed informed consents were obtained from all participants. The data was collected using 7 monopolar EHG sensors, placed around the umbilicus on the abdominal surface. The placement and configuration of electrodes were similar to what was reported in [7] and was chosen post a feasibility study to evaluate the same.. With the intention of not disturbing the clinical workflow and hence the quality of care to the participants, the eighth electrode was not used in order to facilitate the use of CTG (Cardio-Tocograph) sensor simultaneously if required during data collection. Recording was carried out when the cervical dilatation was $3-5 \mathrm{~cm}$. EHG data was recorded for about 30 minutes or at least for about 6-7 contractions. All the 10 participants in the study had normal progress of labour and delivered vaginally. The sensors were arranged as shown in Figure 1. Delsys Trigno wireless EMG system was used to record the EHG signals in this study, and the signals were analyzed using MATLAB.

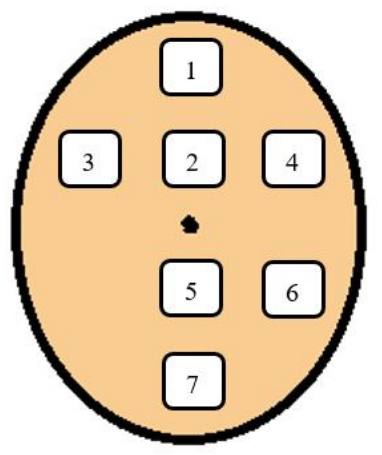

Figure 1: A representation of the location of electrode on the abdominal surface.

The EHG signals from each of the 7 electrodes were quantified and analyzed individually in this study (figure 2). The EHG signal from each electrode was 'Amplitude Demodulated' to extract the slow wave corresponding to the contraction wave. Where, the EHG signal was first band pass filtered using a second order Butterworth filter between the frequency bands of $0.1 \mathrm{~Hz}$ to $0.9 \mathrm{~Hz}$. This step removes the noise added by the fetal movement, motion artefacts, maternal ECG. The second step was to apply a low pass, second order Butterworth filter with a cut off frequency of $0.01 \mathrm{~Hz}$. This step yields an envelope of the signal.

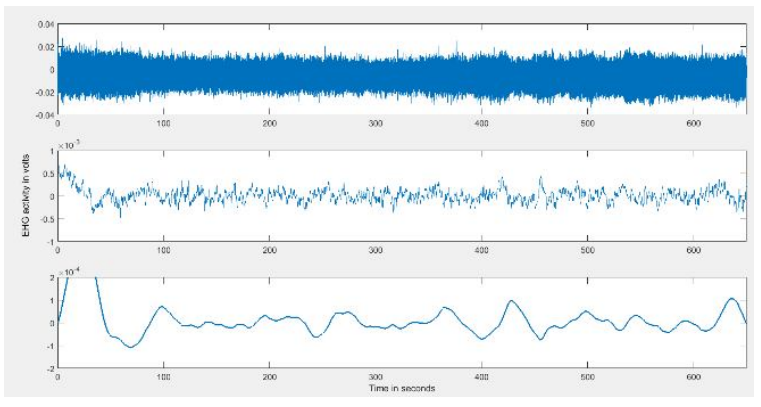

Figure 2: A representation of unfiltered EHG signal at top, EHG signal after first stage of filtering in middle, and envelope of the filtered signal at the bottom.

Two parameters have been used to quantify the Amplitude demodulated signals, namely power of the signal and average peak amplitudes. Power of the signal was calculated as explained. Post extraction of the slow wave from the EHG signal, the Euclidian norm of the signal was calculated. Euclidian norm is also called the vector magnitude and is represented as in the below equation. Where $\mathrm{v}$ represents the vector. This step essentially sums up the amplitude of the entire signal. Squaring and taking the root takes care of the negative values in the signal. Hence the use of RMS (root mean square) or the absolute values on the EHG signal earlier at the filtering stage was refrained from.

$$
|| v||=\sqrt{\sum_{k=1}^{N}\left|v_{k}\right|^{2}}
$$

The power of the signal was then calculated as,

$$
p=\frac{\|v\|^{2}}{\text { length of } v}
$$

For each patient, the average power for the entire recording was calculated for each electrode. Then the electrode wise average power for all the 10 patients was calculated.

The second parameter used in this study is the average peak height of amplitude demodulated wave from each electrode. Peaks were identified based on the thresholds defined for minimum amplitude and distance between two successive peaks. The threshold values were defined for each patient based on a visual inspection of the contractile activity. For 
each patient, electrode wise average peak amplitudes were calculated. Later, electrode wise average peak amplitudes for all 10 patients were calculated.

\section{RESULTS}

The power contained in the EHG signal of each electrode is represented as a scatter plot in figure 3. It is evident from the plot that the power of the signal from electrode 7 is significantly higher compared to the other electrodes. Similarly, in the upper uterine segment it is electrode number 4 which has the highest power.

In case of peak amplitude, the variation between different electrodes are more subtle. However, contrary to the expectations, it was electrode 2 that had the highest peak amplitude. Electrode 1 also had a significant value, comparable to that of electrode 2 . While electrode 7 had the lowest amplitude. This indicates that the contractions strength is maximum below the fundal region when the contractions is at peak.

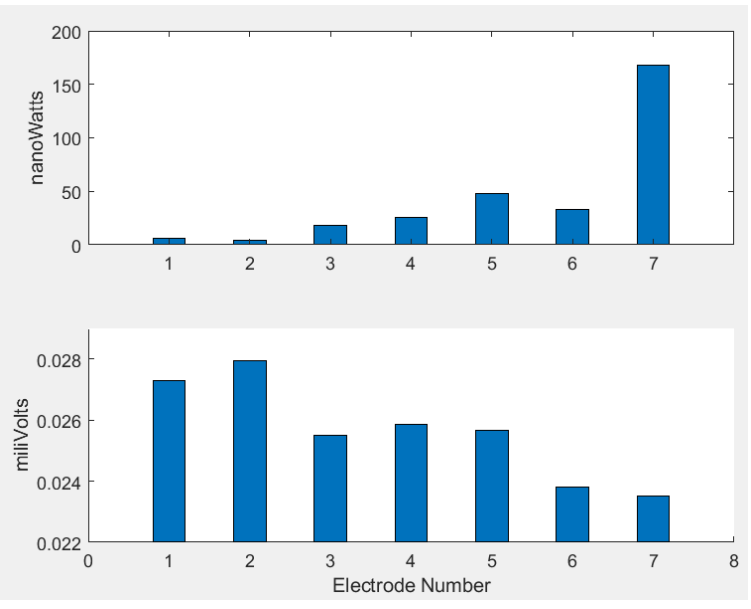

Figure 3: Electrode wise average power for all 10 patients at the top, electrode wise average peak amplitudes for all 10 patients at the bottom.

It can also be observed that, the electrical activity in the sensors placed in line with the umbilicus have consistently had higher power as well as higher amplitudes as compared to the other regions. This information could serve as a template for placement of electrodes for future studies.

\section{DISCUSSION}

These observations can be better understood by the concept of center of uterine activity (CUA) stated in [7]. Uterus is a huge muscle and uterine contraction is a non-stationary signal. The region where the electrical activity initiates the contractions and later spreads to the surrounding cells to sustain the contraction, is considered to be the CUA. Uterine contractions may last anywhere between 20 to 60 seconds. The CUA keeps moving throughout the uterus for the entire duration of the contraction.

Power being higher in the lower uterine segment indicates that the CUA could be in the lower uterine segment for the most part of the contraction, as the contraction strength increases the CUA moves towards the upper uterine segment. These findings reinstates the observations made by [7]. However, since all the participants in our study had normal progress of labor, we cannot confirm their claim that a lack of CUA movement near the fundal region may lead to non-progress.

\section{CONCLUSION}

The study has showed that the region in line with the umbilicus both in the upper uterine segment and lower uterine segment is the region of maximum electrical activity. Studies aiming to reduce the number of electrodes may use this as a template for placement of electrodes, while still being able to collect significant amount of uterine activity.

The observations made could be interpreted as; when the CUA is in the lower uterine segment and the contractile forces are lower, it may be aiding cervical dilatation. But as the contractions strength increases, the CUA moves towards the upper uterine segment to exert the higher force associated with the stronger contraction to aid the descent of the baby.

Power of the electrical activity was observed to be higher in the lower uterine segment as compared to the upper uterine segment. This is observed at around $4 \mathrm{~cm}$ of dilatation, where the primary objective of the contraction at this stage is to aid cervical dilatation. As the labor progresses, the power of the electrical activity may become stronger in the upper uterine segment i.e., dominant fundal activity to aid fetal descent. This transition could occur at the influx point observed on the partogram where the dilatation slows down and the fetal descent accelerates.

\section{ACKNOWLEDGEMENT}

We thank the Dept. of OBG, Dr. TMA Pai Hospital, Udupi, for their immense cooperation during data collection. We thank the Dept. of Physiotherapy, School of Allied Health Sciences, MAHE, and Dr. Senthil Kumaran for providing us the equipment to capture the EHG data. We would also like to thank Dr. Aditi Nair for helping us with the data collection.

\section{REFERENCES}

[1] J. M. Marshall, "Regulation of activity in uterine smooth muscle," Physiol. Rev. Suppl., vol. 5, pp. 213-227, Jul. 1962. 
[2] C. Y. Kao, "Electrophysiological Properties of Uterine Smooth Muscle," SpringerLink, pp. 403-454, 1989.

https://doi.org/10.1007/978-1-4684-5589-2_14

[3] D. Devedeux, C. Marque, S. Mansour, G. Germain, and J. Duchêne, "Uterine electromyography: A critical review," Am. J. Obstet. Gynecol., vol. 169, no. 6, pp. 1636-1653, Dec. 1993. https://doi.org/10.1016/0002-9378(93)90456-S

[4] T. Y. EULIANO et al., "Monitoring uterine activity during labor: a comparison of three methods," Am. J. Obstet. Gynecol., vol. 208, no. 1, pp. 66.e1-66.e6, Jan. 2013.

https://doi.org/10.1016/j.ajog.2012.10.873

[5] M. P. G. C. Vinken, C. Rabotti, M. Mischi, and S. G. Oei, "Accuracy of frequency-related parameters of the electrohysterogram for predicting preterm delivery: a review of the literature," Obstet. Gynecol. Surv., vol. 64, no. 8, pp. 529-541, Aug. 2009. https://doi.org/10.1097/OGX.0b013e3181a8c6b1 [6] J. Garcia-Casado, Y. Ye-Lin, G. Prats-Boluda, J. Mas-Cabo, J. Alberola-Rubio, and A. Perales, "Electrohysterography in the diagnosis of preterm birth: a review," Physiol. Meas., vol. 39, no. 2, p. 02TR01, 262018.

https://doi.org/10.1088/1361-6579/aaad56

[7] T. Y. Euliano, D. Marossero, M. T. Nguyen, N. R. Euliano, J. Principe, and R. K. Edwards, "Spatiotemporal electrohysterography patterns in normal and arrested labor," Am. J. Obstet. Gynecol., vol. 200, no. 1, pp. 54.e1-7, Jan. 2009.

https://doi.org/10.1016/j.ajog.2008.09.008

[8] B. Vasak et al., "Uterine electromyography for identification of first-stage labor arrest in term nulliparous women with spontaneous onset of labor," Am. J. Obstet. Gynecol., vol. 209, no. 3, pp. 232.e18, Sep. 2013.

https://doi.org/10.1016/j.ajog.2013.05.056

[9] B. Vasak et al., "Identification of first-stage labor arrest by electromyography in term nulliparous women after induction of labor," Acta Obstet.
Gynecol. Scand., vol. 96, no. 7, pp. 868-876, Jul. 2017.

https://doi.org/10.1111/aogs.13127

[10] A. Shmygol, A. M. Blanks, G. Bru-Mercier, J. E. Gullam, and S. Thornton, "Control of uterine $\mathrm{Ca} 2+$ by membrane voltage: toward understanding the excitation-contraction coupling in human myometrium," Ann. N. Y. Acad. Sci., vol. 1101, pp. 97-109, Apr. 2007.

https://doi.org/10.1196/annals.1389.031

[11] R. K. Riemer and M. A. Heymann, "Regulation of Uterine Smooth Muscle Function during Gestation,” Pediatr. Res., vol. 44, no. 5, pp. 615-627, Nov. 1998. https://doi.org/10.1203/00006450-199811000-00001 [12] R. E. Garfield and W. L. Maner, "Physiology and Electrical Activity of Uterine Contractions," Semin. Cell Dev. Biol., vol. 18, no. 3, pp. 289-295, Jun. 2007.

https://doi.org/10.1016/j.semcdb.2007.05.004

[13] K. Horoba, J. Jezewski, J. Wrobel, and S. Graczyk, "Algorithm for detection of uterine contractions from electrohysterogram," in 2001 Conference Proceedings of the 23rd Annual International Conference of the IEEE Engineering in Medicine and Biology Society, 2001, vol. 3, pp. 2161-2164 vol.3.

[14] V. Shulgin and O. Shepel, "Electro hysterographic signals processing for uterine activity detection and characterization," in 2014 IEEE 34th International Scientific Conference on Electronics and Nanotechnology (ELNANO), 2014, pp. 269-272. https://doi.org/10.1109/ELNANO.2014.6873918

[15] J. Jezewski, K. Horoba, A. Matonia, and J. Wrobel, "Quantitative analysis of contraction patterns in electrical activity signal of pregnant uterus as an alternative to mechanical approach," Physiol. Meas., vol. 26, no. 5, pp. 753-767, Oct. 2005. https://doi.org/10.1088/0967-3334/26/5/014 